POS $\quad \begin{aligned} & \text { PROCEEDINGS } \\ & \text { OF SCIENCE }\end{aligned}$

\title{
Holographic Duals of 2+1d QFTs with Minimal SUSY with Massive Flavours.
}

\author{
Niall T. Macpherson* \\ Department of Physics, Swansea University, Singleton Park, Swansea SA2 8PP, United Kingdom \\ E-mail: pymacphersoneswansea.ac.uk
}

The dual to $\mathscr{N}=1 \mathrm{SYM}$ in $3 \mathrm{~d}$ is generalised to accommodate massive fundamental matter. This is achieved with a profile for source D5 branes and gives rise to a singularity free IR. A solution technique is then applied which generates the $G_{2}$ structure equivalent of baryonic branch of Klebanov-Strassler with an additional scale mediated by the profile.

Proceedings of the Corfu Summer Institute 2012

September 8-27, 2012

Corfu, Greece

*Speaker. 


\section{Introduction}

An important development in the gauge-gravity correspondence [1] was the technique of adding smeared source branes. This enabled the construction of holographic duals to QFT's with fundamental matter in the Veneziano limit, where $N_{c}, N_{f} \rightarrow \infty$ with $N_{c} / N_{f} \sim 1$ [2]. And so it was made it possible to move out of the probe limit and construct tractable gravity duals to field theories of phenomenological interest. In its original form the method produced duals to gauge theory with massless flavour and many interesting results were found. However these backgrounds generically suffered from unphysical IR singularities caused by the infinite number of flavour branes reaching the origin.

This IR pathology can be solved if a profile is introduced for the flavour branes $N_{f} \rightarrow N_{f} P$ as in Fig 1. Then the dual QFTs have massive flavour $[3,4]$ and the profile is interpreted as follows. In UV, where $r>>m_{Q}^{2}$, the flavours are effectively massless and so $P=1$. In the IR, where $r<<m_{Q}^{2}$, the flavours have been integrated out and so $P=0$. Finally the intermediate region is where the effect of quarks running in loops is becoming increasingly noticeable as the energy increases.

Another development that is showing increasing utility is solution generating techniques, the principal reason being that finding solutions to SuGra is hard. The idea is that one starts from a relatively simple SuGra solution that may not be dual to anything very interesting or may contain pathologies. A solution generating would then be applied to this mapping to a SuGra solution with a richer flux structure, improved metric, and/or fewer pathologies. This more complicated theory would be solved in terms of the simpler one which is already known.

A well understood example of such a technique was that has born much fruit was introduced by Maldacena and Martelli [8]. It is a U-duality (or $S U(3)$ rotation) which takes a deformation [2] of the Maldacena-Nunez solution [10] as its "seed" and maps it to, the baryonic branch [11] of, Klebanov-Strassler [12]. The seed solution is constructed from D5 branes that wrap a 2-cycle inside the conifold and supports only a RR 3-form and dilaton. It is only a good dual to a 4d QFT in the IR as the branes unwrap as one flows towards the UV. The rotated solution supports $F_{3}, F_{5}$ and $H$ and gives the dual QFT a UV completion.

The purpose of this short note is to review some recent developments in holographic duals to 3d theories with minimal SUSY that are closely related to what is described above. The SuGra solution dual to $3 \mathrm{~d}$ SYM with $\mathscr{N}=1$ SUSY and unquenched massive flavours was constructed in [5]. This includes a source D5 profile which provides an interpolation between the MaldacenaNastase solution [6] in the IR and its massless flavour generalisation [7] in the UV. It is constructed from D5 branes that wrap a 3-cycle inside a $G_{2}$ structure manifold and supports a dilaton and $F_{3}$. As shown in [5] and expanded upon in [9], it is possible to apply a $G_{2}$ structure rotation [13] to this solution. The result is a type-IIA solution that is the $G_{2}$ analogue of the Baryonic branch of Klebanov-Strassler generalised to include an intermediate scale mediated by the profile. This solution contains D3,D4 and NS5 branes and has a metric that is asymptotically $A d S_{4} \times Y$ where $Y$ is the metric at the base of a $G_{2}$ cone. In fact, as with the $S U(3)$ structure case [15], it is only possible to have an intermediate scale and desirable asymptotics when one take the source NS5 brane profile to be of the second type in Fig. 1.

The note is contains the following material:

- Section 2 contains a review of the relevant SuGra backgrounds constructed in [5]. 

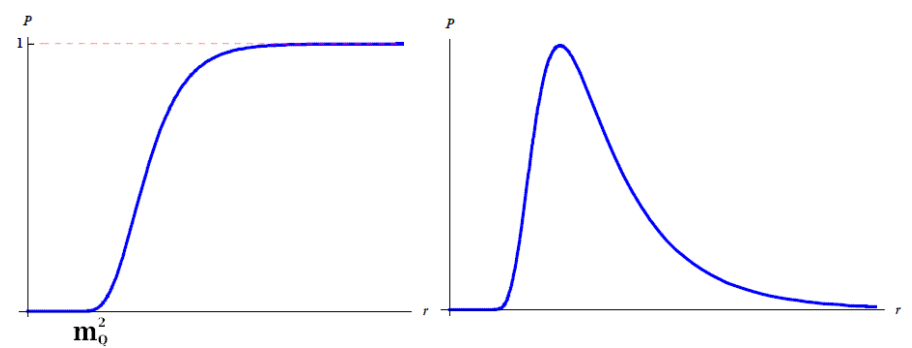

Figure 1: Brane Profiles: The left panel shows a typical profile for massive flavours where the point that the profile becomes non zero give a measure of the quark mass. The right panel shows a profile which keeps $A d S$ asymptotics after rotation.

- Section 3 presents some results for 3d SYM with minimal SUSY and massive dynamical flavours.

- Section 4 reviews some of the results of [9] about the field theory dual to the solution after rotation.

- Finally Section 5 closes with some further comments on why these solutions are interesting and possible future directions.

\section{The SuGra Setup}

The generalised Maldacena-Nastase solution of [5] is a Type-IIB SuGra solution on a $G_{2}$ structure manifold with topology $\mathbb{R}^{1,2} \times \mathbb{R} \times S^{3} \times S^{3}$. Its metric is given in string frame by:

$$
d s^{2}=e^{\phi}\left(d x_{1,2}^{2}+d r^{2}+\frac{e^{2 q}}{4}\left(\sigma^{i}\right)^{2}+\frac{e^{2 g}}{4}\left(\omega^{i}-\frac{1}{2}(1+w) \sigma^{i}\right)^{2}\right)
$$

where the functions $h, g, w$ and the dilaton $\phi$ depend only on the radial coordinate $\mathrm{r}$ and $\sigma^{i}, \omega^{i}$ are 2 sets of $S U(2)$ left invariant 1-forms. The background contains D5 branes which wrap the 3-cycle $\sigma^{i}=\omega^{i}$ and supports a RR 3-form $F_{3}$. There are also source D5 branes which depends on a brane profile $P$. These induce an explicit source that enters into the solution through a violation of the Bianchi identity:

$$
d F_{3}=N_{f} \Xi_{4}
$$

where $\Xi$ is is the smearing form which depends on $P$ and $P^{\prime}$ and is related to the codimensions of the source D5 brane embedding.

The various functions introduced above obey a first order set of coupled differential equations defined by the BPS relations for $G_{2}$ structure backgrounds [16]. When solved these give two distinct types of solution, an asymptotically linear dilaton solution and one with and asymptotically constant dilaton (see Fig. 2). The latter is incompatible with a global flavour symmetry as any putative flavour states are non normalisable. Thus the results about the $3 \mathrm{~d} \mathscr{N}=1 \mathrm{SYM}$ coupled to massive flavour discussed in the next section are obtained from the asymptotically linear dilaton solutions. In both cases the wrapped D5 branes which which make up the geometry unwrap as one 


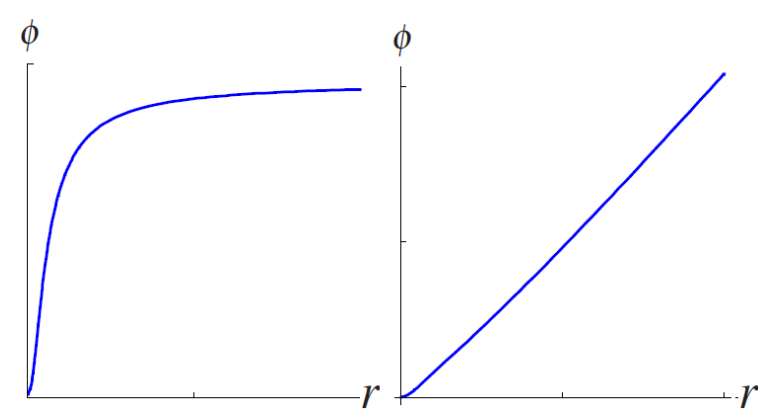

Figure 2: Two types of SuGra solution are characterised by a dilaton which either tends to a constant or becomes linear towards the UV. The first is compatible with a global flavour symmetry while the second with a gauged flavour symmetry.

flows towards the UV so the effective 3d field theory living on the D5 branes becomes manifestly $6 \mathrm{~d}$ at high energies.

The constant dilaton solutions are what are required for the $G_{2}$ structure rotation of [13]. The rotation maps an unwarped type-IIA solution with NS5 branes to a more complicated type-IIA solution. The first step is to make use of s-duality under which:

$$
g_{\mu \nu} \rightarrow e^{-\phi} g_{\mu \nu} ; \quad F_{3} \rightarrow H_{3} ; \quad \phi \rightarrow-\phi ;
$$

One is thus transformed form the type-IIB RR sector into the common NS sector of type-II SuGra. The rotation procedure is equivalent to a lift to M-theory and an infinite boost along $x^{11}$ before a reduction back to type-IIA followed by two T-dualities along the spatial field theory directions. This can be described in terms of the brane content at each step:

$$
N S 5 \rightarrow M 5 \rightarrow M 5, p_{K K} \rightarrow N S 5, D 0, D 2 \rightarrow N S 5, D 2, D 4
$$

The new geometry contains both both D2 and D4 branes which support $F_{4}$, fractional D2 branes that support $H_{3}$ and source NS5 branes. The rotation generates additional warp factors in the metric which is now given by:

$$
\begin{aligned}
d s^{2} & =\frac{1}{\sqrt{h}} d x_{1,2}^{2}+\sqrt{h}\left(d r^{2}+\frac{e^{2 q}}{4}\left(\sigma^{i}\right)^{2}+\frac{e^{2 g}}{4}\left(\omega^{i}-\frac{1}{2}(1+w) \sigma^{i}\right)^{2}\right) \\
h & =1-e^{2\left(\phi_{\infty}-\phi\right)}
\end{aligned}
$$

Provided that new scale is governed by a profile that dies off in the UV, $h$ makes the geometry asymptotically $A d S$. The rotated solution is dual to a gauge theory that is UV complete, the internal space remains finite for all $0 \leq r<\infty$ so as on flows toward the UV the NS5 branes do not unwrap. Note also that the dilaton is modified by the rotation:

$$
e^{2 \hat{\phi}}=e^{2\left(\phi-\phi_{\infty}\right)} \sqrt{h}
$$

This happens because the dilaton couples to the D2 branes that are generated. 


\section{On 3d $\mathscr{N}=1$ SYM with unquenched Massive flavour}

The Type-IIB solution of the previous section with asymptotically constant dilaton is dual in the IR to $3 \mathrm{~d} \mathscr{N}=1 \mathrm{SYM}$ with Gauge group $S U\left(N_{c}\right)$ and flavour group $S U\left(N_{f}\right)^{1}$. The theory also contains a Chern Simons term of level $\mathrm{k}$ which can extracted from the gravity side via a Probe D5 brane calculation[6]. The brane is extended along the field theory directions and wraps one of the $S^{3}$ 's. It is possible to show $[6,5]$ that the level is given by:

$$
k=\frac{1}{4 \pi^{2}} \int_{s^{3}} F_{3}-\tilde{k}=\frac{N_{c}}{2}+\frac{3 N_{f}}{4}(C-1) P
$$

where it has been implicitly assumed that $m_{q}^{2}<<m_{K K}^{2}$ so that the theory living on the D5 branes can be thought of as being effectively $3 \mathrm{~d}$ over a range of $r$ where $P \sim 1$. A shift in the level by $\tilde{k}$ has also been included which is induced when the $6 \mathrm{~d} \mathrm{KK}$ modes are integrated out. Clearly $k$ is only quantised everywhere for $C=1$, however if one thinks of the interpolation region where $P \neq 0,1$ as an approximation to a jump which smooths out the geometry, the profile can be thought of as inducing a shift in $P$ as one flows from the UV to the IR.

The gauge coupling of the theory can be extracted from a probe D5 brane which wraps the cycle $\sigma^{i}=\omega^{i}$ [7]. As explained in [5] for $N_{c} \geq 2 N_{f}$ the gauge coupling is consistent with confinement in the IR and asymptotic freedom in the UV while for $N_{c} \leq 2 N_{f}$ the theory appears to develop a Landau pole in the $\mathrm{UV}^{2}$.

A Wilson loop study can be performed from the gravity side by calculating the area of a minimal surface that extends from the UV, where it ends on the contour of the loop, and extends along $\mathrm{r}$ towards the IR [17]. A rectangular loop gives the potential $E(L)$ between 2 infinitely massive quarks separated by a distance $L$. It can be extracted, as in [5], from the NG action of a probe string. As shown in Fig. 3, when we take the quark mass to be sufficiently large, there is a smooth transition between confining unflavoured behaviour in the IR and power law flavoured behaviour in the UV.

\section{On the field theory dual to the Asymptotically $A d S$ Solution.}

The rotated solution is generated in a way entirely analogous to the method by which Maldacena and Martelli generate the Baryonic branch solution. Thus one expect the field theory dual to be similar to the baryonic branch of Klebanov-Strassler. In particular it is likely a 2-node quiver of the type $S U(N) \times S U(M)$ exhibiting both a duality and Higgsing cascade as one flows toward the IR, like [15]. However with the computational machinery currently available for $G_{2}$ structure backgrounds this is difficult to show explicitly. What is clear is that the NS5 Maxwell charge is quantised while there is a page charge for D2 that is defined up to quantised shifts under large gauge transformations. This is at least suggestive of a duality cascade like that of [18].

It is possible to define several coupling for the dual field theory with various probe branes. Two that exhibit the likely dynamics ${ }^{3}$ of the dual QFT are $g_{1}$, given by a probe D2 brane on $\left(t, x^{1}, x^{2}\right)$

\footnotetext{
${ }^{1}$ Actually the smearing of the source branes breaks this to $U(1)^{N_{f}}$

${ }^{2}$ This second result should be taken with caution. An improved radial variable was introduced in [9] and it is possible that a Seiberg like duality, not manifest in the radial coordinate of [5], will play a role when $N_{c}<2 N_{f}$. This would make the situation rather more similar to 4d SCQD, as one might expect.

${ }^{3}$ or at least this the most attractive interpretation of the dynamics.
} 

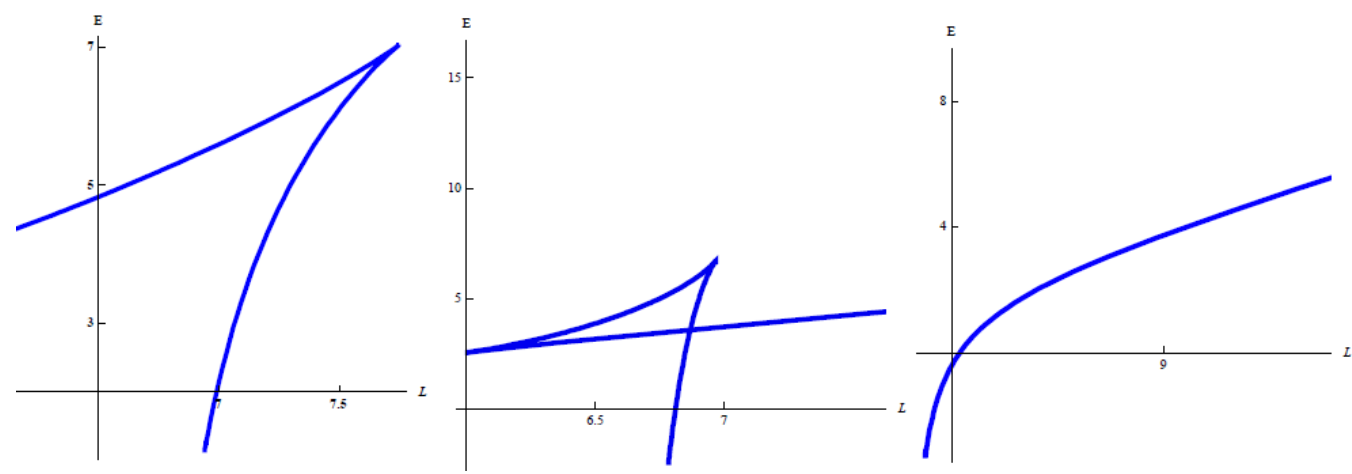

Figure 3: Inter-quark potential extracted from rectangular Wilson loops. The left panel show $m_{Q}^{2}=0$, this has $P=1$. The unphysical cusp is a result of the flavour singularity in the IR. The middle panel shows $0<m_{Q}^{2}<<1$, there is a first order phase transition between between confining, unflavoured, behaviour in the IR and power law behaviour in the UV. The last panel shows the same with a larger value of $m_{q}^{2}$, the phase transition is now smooth.

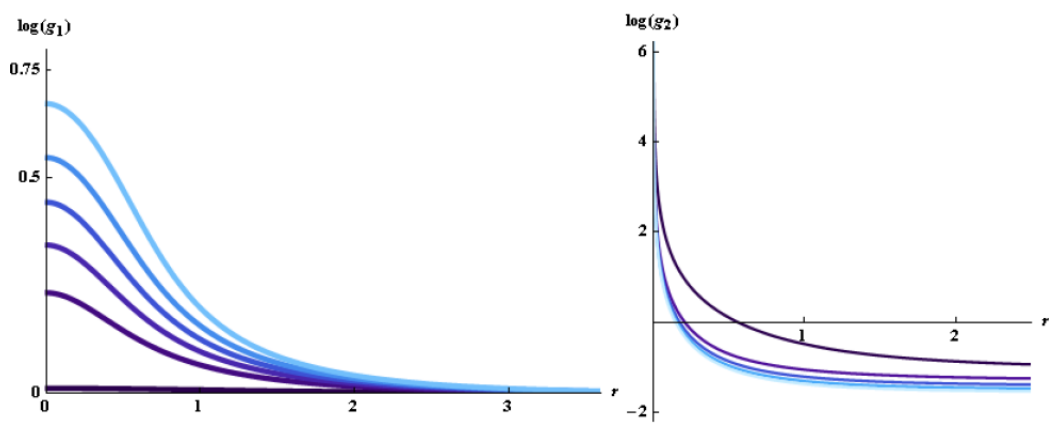

Figure 4: Two coupling of the dual gauge theory. In each case purple to blue indicates an increase in the ratio $N_{f} / N_{c}$

and $g_{2}$, given by a probe $\mathrm{D} 4$ on $\left(t, x^{1}, x^{2}, S^{2}\right)$. These are plotted in Fig. 4.

The coupling $g_{2}$ is consistent with confinement in the IR and dilation invariance in the UV, it cannot be full conformal invariance as this must be broken by the $r$ dependence of the dilaton there. The behaviour is confirmed in [9] with a Wilson loop study similar to that described in the previous section. This is extremely attractive behaviour for a QFT with a well defined gravity dual, so it is desirable to interpret this coupling as describing the fully dynamics of the theory. In light of this $g_{1}$ might appear strange, however one needs to appreciate that Maxwell couplings in $3 \mathrm{~d}$ are dimensionful. This means that when a Chern-Simons term is present, the Maxwell coupling can freeze out in the IR where a confining Chern-Simons theory becomes dominant. For this reason it seems likely that $g_{1}$ is a Maxwell type coupling and its IR behaviour is signalling the presence of a Chern-Simons term.

\section{Conclusions}

In this short note the two closely related type-II $G_{2}$ structure solutions constructed in $[5,9]$ 
were reviewed. These are both dual to confining $3 \mathrm{~d}$ gauge theories with minimal SUSY and so it would be interesting to further analyse their dynamics, particularly as QFTs of this type have large evaded field theoretical analysis.

The construction of the dual to 3d SYM with massive flavour is important because the massless flavour dual of [7] did not have a solution to the BPS equations in terms of a semi analytic IR expansion. As the wrapped D5 branes are only effectively $3 \mathrm{~d}$ in the IR this is a particularly acute pathology and is cured by the addition of the flavour profile. This potentially makes possible the calculation of several interesting quantities in the theory such as the glue ball and Meson spectrum's.

The rotated background is asymptotically $A d S_{4}$ and it is likely that it will provided a fruitful arena for holographic renormalisation group techniques in $3 \mathrm{~d}$. It is particularly interesting to see that the couplings are consistent with confinement in the IR and and dilation invariance in the UV. This is clear if one considers the fact that there are no regular holographic duals to gauge theories that both confine and flow to an exact conformal fixed point in the UV. Unfortunately the dual gauge theory is still little understood but what is know does indicate that there is interesting dynamics waiting to be understood.

\section{References}

[1] J. M. Maldacena, Adv. Theor. Math. Phys. 2 (1998) 231 [hep-th/9711200].

[2] R. Casero, C. Nunez, A. Paredes and, Phys. Rev. D 73 (2006) 086005 [hep-th/0602027].

[3] F. Benini, F. Canoura, S. Cremonesi, C. Nunez, A. V. Ramallo and , JHEP 0702 (2007) 090 [hep-th/0612118].

[4] E. Conde, J. Gaillard, A. V. Ramallo and, JHEP 1110 (2011) 023 [arXiv:1107.3803 [hep-th]].

[5] N. T. Macpherson, JHEP 1206 (2012) 136 [arXiv:1204.4222 [hep-th]].

[6] J. M. Maldacena, H. S. Nastase and , JHEP 0109 (2001) 024 [hep-th/0105049].

[7] F. Canoura, P. Merlatti, A. V. Ramallo and , JHEP 0805 (2008) 011 [arXiv:0803.1475 [hep-th]].

[8] J. Maldacena, D. Martelli and , JHEP 1001 (2010) 104 [arXiv:0906.0591 [hep-th]].

[9] N. T. Macpherson, arXiv:1301.5178 [hep-th].

[10] J. M. Maldacena, C. Nunez and , Phys. Rev. Lett. 86 (2001) 588 [hep-th/0008001].

[11] A. Butti, M. Grana, R. Minasian, M. Petrini, A. Zaffaroni and , JHEP 0503 (2005) 069 [hep-th/0412187].

[12] I. R. Klebanov, M. J. Strassler and , JHEP 0008 (2000) 052 [hep-th/0007191].

[13] J. Gaillard, D. Martelli and, JHEP 1105 (2011) 109 [arXiv:1008.0640 [hep-th]].

[14] J. Gaillard, D. Martelli, C. Nunez, I. Papadimitriou and , Nucl. Phys. B 843 (2011) 1 [arXiv:1004.4638 [hep-th]].

[15] E. Conde, J. Gaillard, C. Nunez, M. Piai, A. V. Ramallo and , JHEP 1202 (2012) 145 [arXiv:1112.3350 [hep-th]].

[16] J. Gaillard, J. Schmude and , JHEP 0901 (2009) 079 [arXiv:0811.3646 [hep-th]]. 
[17] J. M. Maldacena, Phys. Rev. Lett. 80 (1998) 4859 [hep-th/9803002].

[18] F. Benini, F. Canoura, S. Cremonesi, C. Nunez, A. V. Ramallo and , JHEP 0709 (2007) 109 [arXiv:0706.1238 [hep-th]]. 